\title{
Germanité et amitié : trois modèles d'entraide
}

\section{Nicolas Herpin et Jean-Hugues Dechaux}

\section{OpenEdition}

\section{Journals}

Édition électronique

URL : http://journals.openedition.org/ress/400

DOI : $10.4000 /$ ress.400

ISSN : 1663-4446

\section{Éditeur}

Librairie Droz

\section{Édition imprimée}

Date de publication : 1 mars 2004

Pagination : $179-190$

ISBN : 2-600-00941-8

ISSN : 0048-8046

Référence électronique

Nicolas Herpin et Jean-Hugues Dechaux, «Germanité et amitié : trois modèles d'entraide », Revue européenne des sciences sociales [En ligne], XLII-129 | 2004, mis en ligne le 05 novembre 2009, consulté le 19 avril 2019. URL : http://journals.openedition.org/ress/400 ; DOI : 10.4000/ress.400 


\section{GERMANITÉ ET AMITIÉ: TROIS MODÈLES D'ENTRAIDE}

L'entraide recouvre d'innombrables activités mais implique aussi de la confiance et suscite des aspirations. Les instituts statistiques qui comptabilisent les échanges gratuits entre foyers observent les biens offerts ou reçus et des services rendus. De fait, une partie de la production domestique - fruits ou légumes récoltés dans son jardin, transport de personnes dans son véhicule, cuisine et ménage, garde d'enfant ou soins aux malades, bricolage, conseil pour financer l'acquisition d'un logement, pour entreprendre une procédure de divorce, pour l'orientation scolaire d'enfant ou d'adolescent - est «consommée » à l'extérieur du foyer producteur (Crenner, 1999). Une enquête fondée sur une quarantaine d'entretiens ${ }^{1}$ confirme bien l'existence de ces prestations, analogues à celles qu'enregistrent les nomenclatures des enquêtes Budget des Familles. Mais l'entraide entre foyers et entre personnes a des objectifs d'une autre nature, beaucoup plus difficiles à saisir. Ils sont l'objet principal de cette étude monographique sur les relations interpersonnelles.

Pour certains alea de l'existence - le divorce ou des échecs scolaires des enfants - aucune offre marchande de protection n'existe. De plus, lors de nombreux autres événements malheureux, même si les primes financières prévues sont versées, la plupart des dommages ne sont pas intégralement compensés. Par exemple, la caisse d'assurance maladie ne prend pas en compte la douleur des proches; les indemnités de chômage ou le RMI ne permettent pas au chômeur ou au Rmiste d'obtenir des prêts bancaires si aucune personne de son entourage ne se porte garante.

Un troisième type d'entraide échappe encore davantage aux nomenclatures conçues pour mesurer le niveau de vie. La conduite de l'existence est affectée par les contacts entre proches - notamment avec les germains (frères et sœurs) et avec les amis. Plus encore que la contemplation esthétique et la participation artistique (Habermas, 1980), le dialogue et la communication interpersonnelle font découvrir un autre point de vue sur le «monde vécu». L'ouverture à l'autre est en ellemême une fin quand elle est source de réconfort, de compassion, de compréhension, d'écoute et de formation de soi par soi. L'intersubjectivité n'est

Ces entretiens ont été réalisés en France en 2001 auprès de personnes entre 30 et 55 ans, ayant au moins un frère ou une sœur, vivant en couple et dont le conjoint a au moins un frère ou une sœur. Cet article est une partie de l'étude sur les liens de germanité à l'âge adulte, étude d'ensemble dirigée par J-H Déchaux et financé par la Mire (ministère des affaires sociales). A ces quarante entretiens, s'ajoute l'exploitation statistique d'une enquête Insee de 1997 portant sur le réseau de parenté et l'entraide. 
pas non plus sans conséquence sur les projets personnels. Cette mise à l'épreuve ébranle des certitudes et affermit des convictions personnelles.

Plus encore que la germanité, l'amitié n'est-elle pas au premier chef concernée par ce dernier type de service? En général, on suppose que les formes de l'entraide ne se distribuent pas de la même façon selon la nature du lien. On n'attend pas des amis ce qu'on attend des frères et sœurs. L'article montre que cette conception spécialisée de l'entraide existe bien. Mais il ajoute deux autres modèles identifiés à partir des entretiens effectués. Plusieurs conceptions de l'entraide entre proches coexistent donc dans les sociétés occidentales modernes. La conclusion s'interroge sur cette coexistence.

\section{LES SÉPARATEURS}

Les séparateurs font de la germanité une relation absolument distincte de l'amitié. Chacune a son principe propre. Le réseau des fréquentations classe séparément les amis d'une part et les frères et sœurs d'autre part. Les activités, tout comme la nature des échanges, diffèrent profondément selon la nature du lien.

Entre germains, la relation interpersonnelle est indestructible. La fratrie peut être le théâtre de conflits. Les ruptures, même durables, ne sont jamais définitives. Ego (homme, 42 ans, cadre de banque) a vu se coaliser contre lui son frère, sa sæur sous la houlette de leur mère au moment du divorce de cette dernière avec le père, accusé d'alcoolisme. Certes, Ego est bien accueilli par sa belle famille. Mais «une famille, ça ne se remplace pas. Je dirai que ma famille restera ma famille. La famille de ma femme ne se substitue pas à celle que j'ai perdue». Il prend contact avec ses cousins et ses oncles paternels et maternels pour rétablir par leur médiation les liens avec son frère et sa sœur, plus accessibles selon lui que leur mère.

Amitié et germanité sont fondées sur deux principes différents. La germanité est une relation de sang et c'est pourquoi elle ne peut être rompue. Elle renvoie à une symbolique du sang partagé qui fait du germain un proche présumé (Déchaux, 1997). A l'inverse, l'amitié, relation volontaire, est éphémère. Sa pérennité dépend du bon vouloir des deux protagonistes. Ego (homme, 48 ans, employé de bureau) fait cette comparaison: «Un frère tu peux le garder à vie. Tu es obligé: tu le garderas à vie. Tu peux te fâcher et puis 10 ans après tu vas te réconcilier. Un ami, ça peut être très fort. Tu peux avoir des liens peut-être plus forts même qu'avec tes frères et sœurs. Moi j' ai eu des amis comme ça. Puis après les liens se sont distendus. C'est pas qu'on est fâché, mais on ne se voit plus».

Ces principes différents ont des implications sur la communication. Entre amis, les échanges sont plus spontanés, plus sincères, plus «vrais ». D’où le caractère souvent passionné des discussions. Entre frères et sœurs, on ne peut pas se permettre les violences verbales qu'on s'autorise entre amis. Ego ( homme, 53 ans, agent technique) compare le repas de famille avec les beaux-frères, dont pourtant certains sont presque devenus des amis, et le repas avec les amis de pétanque: "Quand tu manges avec la famille, c'est pas comme quand tu manges avec les amis de pétanque (...). Là-bas, tu te défoules. Exactement, tu racontes des bêtises. Des gros mots, Ouh là! A la pétanque, y'en a des gros mots (...) Putain' con (...). Encaga. Tu vois c' est du patois, ça. Et après, tu vas boire un coup 
et tout s'arrange. Tu t'engeules aussi. Mais c'est des disputes de jeu. Alors que quand tu t'engueules avec la famille, Oh, là, là, c'est pas marrant».

La germanité implique plus de retenue mais aussi de dissimulation. Il faut avoir à l'esprit les conséquences de ce que l'on dit, notamment sur l'ensemble de la fratrie. Il faut savoir tenir sa langue, laisser les discussions qui pourraient raviver les clivages et mettre en péril l'unité de la fratrie. Ego (homme, 42 ans, chef de projet informatique) a mobilisé sa fratrie pour soutenir un de ses frères "qui s'était laissé piégé par l'alcool». La démarche commune a porté ses fruits. Les relations restent cependant tendues dans cette fratrie où la mère n' a pas de bonnes relations avec les pièces rapportées. Avec certains frères, «il faut éviter de parler éducation des enfants, de leur réussite scolaire. Il faut éviter de parler de vacances et d'argent. C'est vrai qu'après, il ne reste pas grand chose. On reste sur ses gardes.» Il est rare que le soutien moral entre germains aille au-delà d'une attitude de prévenance et d'attention polie. La confidence, a fortiori celle qui concerne la vie de couple, est réservée aux amis.

$\mathrm{Si}$, avec les amis, la communication va plus loin qu'avec les frères et sœurs, c'est sans doute en partie parce qu'on connaît trop bien ses germains. L'amitié, au contraire, est une expérience d'intersubjectivité. On s'y révèle à autrui, mais aussi on y découvre un autre point de vue sur l'existence. Ego (homme, 42 ans, comptable dans un centre de gestion agricole) dit: «Avec un ami, il n'y a pas de censure, il n'y a pas de barrières. Il y a moins d'arrière-pensées. C'est plus direct comme relation. Il n'y a pas la trame des conséquences de la parole». Avec les amis, "on est plus habitué à parler de choses fondamentales. C'est pas la pluie et le beau temps quoi!» comme cela arrive souvent dans les réunions de famille.

La fréquentation séparée de la fratrie et des amis est une conséquence de l'opposition des deux types de communication. Ego (femme, 56 ans, assistance sociale) et son conjoint (chef d'atelier à la Sncf) sont issus de deux familles de gauche, l' une socialiste, l' autre communiste. Les deux fratries ont de bonnes relations et leurs membres sont toujours contents de se retrouver. Mais pour Ego les amis restent une relation plus intellectuelle: "Je pense qu' au niveau des discussions, on a une ouverture, une élaboration de la pensée qui est d'un plus haut degré avec les amis. Les discussions sont plus... argumentées. Y'a plus d'échanges, voilà.» Ego et son conjoint évitent de mélanger les amis et la famille. Les activités, notamment celles de loisir, entreprises avec les uns ou les autres sont de nature différente. Ego et son conjoint sortent au théâtre ou dans des soirées musicales beaucoup plus volontiers avec des amis qu' avec de la famille. Il leur arrive aussi de partir en vacances avec des amis, ce qui ne se produit pas avec les frères ou les sœurs.

Cette gestion séparée conduit parfois à des situations qui sont explicitement perçues comme peu satisfaisantes. Certaines fratries comportent de telles obligations que le ménage n'a pas les ressources (en temps libre surtout) pour cultiver à l'extérieur des amitiés. Ego (femme, 49 ans, principale d' un collège) conclut l' entretien en constatant que les fratries des deux côtés sont très nombreuses et très prenantes tant pour elle-même que pour son conjoint: «Si on n' avait pas autant de frères et sours, on aurait peut-être plus d'amis».

En maintenant séparés les amis et la fratrie, on perd les bénéfices de l'interaction entre les deux groupes. Il peut en résulter un isolement non voulu. 
Ego (femme, 47 ans, tient le magasin de son conjoint, artisan tapissier) voit relativement peu sa famille et celle de son conjoint, sauf une sæur de son conjoint qu' elle tient pour une amie. Elle constate le relatif isolement dans lequel se trouve son couple. Le couple a perdu de vue des amis qui, bien que proches à l'époque, n'ont pas été choisis comme parrain ou marraine des enfants. Le couple n'a jamais eu non plus l'idée de les inviter avec leurs propres frères et sœurs. Ego réalise qu' en ne cherchant pas à rapprocher leurs amis de leurs germains, elle a sans doute commis là une erreur qui explique en partie leur solitude actuelle.

En résumé, ce mode de gestion qui sépare les germains et les amis ne met pas en concurrence l'amitié et la germanité. Il juxtapose les activités, les échanges, les attentes et les espoirs, mais aussi les réseaux. On retrouve la distinction classique entre «eux» et «nous», les parents et les autres, à ceci près que les germains ne sont pas nécessairement ceux sur qui on peut compter. La germanité fournit une relation de dernier recours qu'on préfèrerait ne pas avoir à activer, mais dont ceux qui ont une fratrie ne sauraient se priver. L'amitié, en revanche, a le beau rôle: elle libère des routines, y compris de celles, qu'insidieusement et contre ses goûts authentiques ou ses talents cachés, on s'impose à soi-même.

\section{LE GROUPE DES PAIRS}

Dans la gestion des séparateurs, la fratrie à l'âge adulte (celle d'Ego ou celle de son conjoint) est conçue comme un héritage. Les amis en sont tenus à l'écart pour ne pas «polluer» la mémoire de la famille nucléaire d'origine. Les enfants assurent désormais la gestion d'un groupe de parenté effectuée auparavant par leurs propres père et mère. En d'autres termes, les relations de germanité endossent les obligations de la filiation, quand les parents ne sont plus là ou ne sont plus en situation d'animer les relations de filiation.

Le groupe des pairs, quant à lui, se définit comme une organisation primaire alternative à la famille nucléaire (Gans, 1962). Il mélange des copains de jeunesse, des germains, des cousins, des voisins, des condisciples des deux sexes. A l'origine de sa composition, deux critères interviennent: la proximité de l'âge et celle du voisinage. Par la suite, d'autres facteurs relevant davantage des affinités interpersonnelles prennent de l'importance. Mais l'admission reste soumise à l'approbation des autres membres du groupe et le groupe influence la vie sociale de chacun de ses membres.

Certaines fratries, dans le corpus des entretiens, présentent de nombreuses caractéristiques du groupe des pairs, notamment parmi les personnes issues de familles rurales. Ce groupe, dont l'influence morale s'exerce sur la vie entière de tous les membres, est composé: 1) de personnes de la même classe d'âge;2) de certains membres de la fratrie (les plus éloignés par l'âge appartiennent à d'autres groupes de pairs); 3 ) de cousins et d'amis recrutés dans le voisinage (du moment qu'ils sont de la même classe d'âge); 4) d'hommes et de femmes (notamment les conjoints des uns et des autres). La composition peut varier au cours de l'existence, mais inclut toujours des membres de la bande de jeunesse. Les père et mère qui appartiennent eux-mêmes à leur propre groupe de pairs, sont tenus à distance de leurs enfants. Les enfants aussi sont tenus à distance, encouragés à former euxmêmes et de façon précoce, leur propre groupe d'âge. 
En dépit du temps écoulé, la bande de jeunesse n'a pas disparu. Ses membres sont devenus des voisins, des collègues de travail et/ou des partenaires de sport. Ego homme (39 ans, électricien salarié dans une entreprise) a gardé son groupe de copains de jeunesse. Condisciples à l'école primaire, camarades de régiment pendant le service national, ils se retrouvent maintenant dans la même équipe de foot. Ceux de ses frères qui partagent la même passion se sont joints au groupe et tout ce monde se rencontre à l'improviste chez Ego pour discuter en buvant l'apéro lorsqu'il n'y a pas de match.

Les femmes de footeux sont aussi membres du groupe. Ego a connu les frères de son épouse avant de rencontrer cette dernière. Le groupe accueille aussi certains d' entre eux qui ont le même âge qu'Ego. En revanche, ceux de sa propre fratrie qui n'ont pas la même passion sont plutôt mis à distance (tous les frères s'échangent des services de bricolage néanmoins). Les traits physiques qui correspondent à des traits de caractère font coupure au sein de la fratrie: les bagarreurs qui ressemblent au père jouent au foot; les plus conciliants qui ressemblent à la mère ont d'autres goûts dans le domaine des loisirs.

A la différence du modèle du «peer group» élaboré par Gans, les membres de ce groupe de pairs n'ont pas pris leur distance à l'égard de la génération des parents. Les deux familles d'origine sont insérées localement, se connaissent et sont de milieu social équivalent. Les relations d'Ego et de son conjoint avec les ascendants des deux côtés restent fréquentes et fortes notamment à l'occasion des fêtes calendaires: on y danse et y organise des jeux.

Dans certains cas, au contraire, le groupe des pairs nécessite que l'on ait rompu les liens de filiation avec sa propre famille mais aussi avec sa belle-famille. Ego (femme, 38 ans, assistante maternelle) et son conjoint (ouvrier d' usine) vivent en symbiose avec le jumeau du conjoint et la copine de ce dernier. Ego a une sæur avec laquelle elle ne s'entend pas. En revanche, elle est devenue très intime avec la copine du jumeau («sa meilleure amie»). De leur côté, les jumeaux ont rompu avec le reste de leur famille, notamment le reste de leur fratrie (un autre frère et deux sœurs). Les père et mère des jumeaux et les autres membres de leur fratrie sont qualifiés «d'un peu spéciaux», sans plus de précision. La rupture entre les deux familles d'origine se traduit par le fait que Noël n' est pas l'occasion d'une fête de famille. Les deux couples des jumeaux réunissent leurs amis pour Noël.

Ici, le modèle de Gans est moins bien respecté pour ce qui a trait à la formation originelle du groupe. La bande de jeunesse ne constitue pas le socle du réseau amical. Cependant, les deux jumeaux forment bien avec leurs amis une «clique» dont tous les membres se connaissent et se fréquentent tantôt chez les uns, tantôt chez les autres. Ces amis communs sont venus d'horizons différents (l'usine, d'une part, et les responsabilités municipales, d'autre part).

La bande de jeunesse reste l'idéal de la vie sociale quand la fratrie est désunie. Ego (femme, 40 ans, agent des impôts) a rencontré son futur conjoint dans le groupe de jeunesse qu' elle fréquentait avec le frère qui lui était le plus proche par l'âge. Les autres frères plus âgés n' appartenaient pas à ce groupe. Vingt ans plus tard, le groupe continue de se réunir de façon épisodique autour d'un méchoui. Mais le frère avec lequel Ego était proche n'en est plus membre, sa belle-sœur l'entraînant dans sa propre famille.

Ego éprouve de la nostalgie pour la vie collective du groupe de jeunesse. Ni sa fratrie, ni celle de son conjoint ne forme une communauté vivace. Preuve de la 
dislocation de la fratrie, les père et mère d'Ego reçoivent séparément les sousgroupes qui s'entendent parmi les frères et sœurs sans chercher à recoller les morceaux pour former une fratrie unifiée. Ego tente de renforcer les liens, sans beaucoup de succès. Se disant plus proche de ses amis que des deux fratries, elle garde comme modèle de vie sociale celui du groupe des pairs, idéal qui lui reste en tête depuis sa jeunesse.

Parfois il peut être envisagé de restaurer le groupe de pairs. Lorsque le lien de filiation se dévitalise et que les frères qui ont réussi professionnellement pourraient revenir au pays, la restauration prochaine du groupe des pairs devient un scénario crédible. Ego (femme, 40 ans, employée de boulangerie) est issue d'une famille rurale. Dans l'enfance, elle a fréquenté avec ses deux frères un peu plus âgés la même bande et y a rencontré son futur conjoint. La plupart des copains actuels du couple sont issus de cette équipe de foot, du temps de la jeunesse. Autre élément de conformité avec le modèle de Gans, le père d'Ego est décédé et les relations d'Ego avec sa mère sont exécrables. Enfin, bien que sa sœur habite à proximité, Ego ne se sent pas proche affectivement: elle ne lui a pas pardonné une trahison dans la jeunesse (la sœur a révélé à la mère la première expérience sexuelle d'Ego).

Ego aspire et œuvre à la résurrection du groupe de jeunesse. Ses frères qui ont connu une belle réussite socioéconomique (l'un est devenu professeur, l'autre ingénieur) et qui vivent loin du pays d' enfance (l'ingénieur vit en Afrique), restent cependant très proches affectivement de leur sœur. Elle espère qu'avec l'approche de la retraite ils reviendront s' installer à proximité. L'un d' entre eux prospecte pour acheter une maison dans la région. La restauration du groupe des pairs (mais non de la fratrie au complet) est pour elle en chemin.

Le groupe des pairs peut s'élargir à la communauté villageoise quand l'agglomération de résidence est de petite taille et la personne très insérée dans son environnement. Ego (homme, 38 ans, BEP-CAP d'électromécanicien) est né dans son village et a une vie sociale locale extrêmement chargée. Son épouse et lui ont récemment décidé d' un commun accord de refuser les invitations du vendredi soir pour au moins avoir une soirée ensemble par semaine. Les amies de classes de son épouse (institutrice) organisent tous les ans un réveillon pour la nouvelle année. Ses propres amis de classes qui, pour les garçons, sont aussi les membres de sa classe de conscription font une fête tous les ans. Au travail, il y a aussi un petit groupe de collègues qu' il considère comme des amis. Il participe avec des amis, qui sont plutôt ceux de son frère aîné, à un concours de pétanque une fois par an. Avec les collègues élus de la mairie, "on se fait une petite bouffe, des apéros dînatoires».

Ces diverses fréquentations, même si elles donnent lieu à des activités distinctes, regroupent souvent les mêmes habitants du village. Plusieurs cercles sont imbriqués. Ego ne fait pas de distinction forte entre les amis et les membres de sa fratrie. Assurément, il se sent solidaire de son frère et de sa sœur. Il déclare: "s'il arrive quelque chose à mon frère ou à ma sæur... Ah! non, non. Il faut pas y toucher. C'est en terrain protégé.»Il n'est pas convaincu en revanche par la définition courante de l'amitié: "On dit souvent qu'on ne choisit pas sa famille mais on choisit ses amis». Mais pfou!, comme je ne suis pas d' une nature à me confier, je ne fais pas la différence. Peut-être qu' un véritable ami, j' irais tout dire. Mais je n' en ai pas». Avoir un ami, ce serait faire bande à part et s' exclure de la communauté dans laquelle Ego est un personnage influent. 
L'engagement militant peut entraîner, comme sous-produit, la création d'un groupe d'amis sur lesquels on peut compter en toutes circonstances. Ce groupe a des fonctions variées. Par exemple, les relations qu'il peut mobiliser sont mises à profit pour lancer l'entreprise d'un de ses membres. Ego (un homme, 47 ans, agriculteur) est rebelle à l'égard de sa propre famille et de celle de son conjoint. Avec son frère la cassure, qui est ancienne, est de nature politique (le frère, maire de la commune, a accepté l' implantation d'un stockage des déchets nucléaires). Ego a refusé de reprendre l' exploitation agricole de ses beaux-parents (c'est une famille de filles) et a préféré emprunter pour acheter la sienne. Opposé à la mondialisation qui condamne le petit indépendant agricole, il s'est appuyé sur le réseau de ses camarades militants anti-mondialistes pour trouver un financement.

A la différence du modèle de Gans, le groupe politique dont il est un membre actif n' est pas une émanation de l'enfance ou de la jeunesse, mais résulte de l'action militante. Y figurent plus d'amis que de parents. Cependant, un de ses jeunes frères le rejoint car il se révèle avoir les mêmes convictions qu'Ego. La relation de filiation a été en partie dévitalisée dès l' enfance par les conflits entre les pèremère et les grands-parents. Ego a quitté très jeune le foyer familial trop conflictuel et s' est fait employer par un de ses oncles.

En résumé, le groupe des pairs est une entité collective. Le clivage n'est pas entre germains et amis, mais entre membres et non-membres du groupe. Le groupe n'est pas indifférent aux relations de parenté entre certains membres du groupe, notamment dans sa période de formation et au moment où collectivement il se prononce sur l'admission de nouveaux membres. Cependant dans les activités coordonnées de la vie quotidienne, les relations intra-groupe font disparaître cette distinction. Les foyers du groupe de pairs sont ouverts les uns sur les autres de façon homogène. Le groupe offre un «pool» de personnes dont les goûts, les compétences et les ressources sont connus de tous. C'est dans ce cadre restreint que s'effectue l'éventuelle sélection des plus aptes à être mobilisés dans les loisirs, les activités associatives oules activités économiques (coup de main, recommandation, conseils et information, etc.).

\section{LES «RÉSEAUTEURS»}

Les «réseauteurs» gardent le contact avec des fréquentations nombreuses mais qui ne comportent pas nécessairement des relations très intimes. Les plus intimes ne sont pas toujours des amis. On peut en trouver parmi les frères et sœurs ou les beaux-frères et belles-sœurs, mais aussi parmi les pièces rapportées des deux côtés. Ces relations forment parfois des réseaux de personnes qui se connaissent entre elles; mais le plus souvent, il ne s'agit pas d'un groupe d'interconnaissance comme le groupe des pairs soudé par une histoire commune, une cohésion et des liens forts. Ces liens nombreux et faibles sont surtout ceux dont l'utilité éventuelle est de nature économique: ce sont des «introductions» possibles dans les relations d'affaire, des sources de bonnes adresses ou d'informations, etc. L'interconnaissance et l'intimité peuvent être plus poussées pour des relations de loisir: le groupe avec lequel on pratique un sport, celui du bridge, celui avec lequel on part en voyage organisé, on va au théâtre, on organise des dîners chez soi ou des sorties au restaurant. Chaque cercle de composition 
différente a un objectif différent et aucun n'influence de façon prédominante la vie sociale.

Les différents membres du réseau ne sont pas fréquentés avec les mêmes attentes. Cette individualisation de la relation concerne aussi bien la fratrie que les amis ou les simples connaissances. Par conséquent, la distinction entre amitié et germanité s'efface. Ego (femme, 33 ans, professeur en collège) a une vie sociale très active mais organisée comme activité de couple: «il y a les amis de la fac, qu'on revoit deux ou trois fois l'an». Plusieurs autres cercles sont évoqués. Ils sont fréquentés, chacun, de façon séparée. "Je n'ai pas envie de mélanger les amis et les collègues. Mais on peut dire que nous avons un petit réseau d'amis parmi les collègues de travail». Pour ego, les amis sont ceux avec lesquels «on a une communauté de pensée». Parmi ceux-là, le couple est plus intime avec ceux qui «ont des convictions religieuses identiques aux nôtres». Enfin, il y a les personnes du village où ils habitent. Ce sont «des familles qu' on voit par nos engagements paroissiaux, par nos engagements au niveau de l'école, les parents des amis de nos enfants». En raison de la taille du village, ce cercle-là forme un groupe d'interconnaissance. Mais il n'a pas d' influence particulièrement étendue et forte comme l'aurait un groupe de pairs.

Enfin, il y a les deux familles d'origine et notamment les deux fratries, nombreuses. Elles ne sont pas vraiment traitées à part. Certes, «la famille (...) on ne l'a pas choisie. Je ne dirais pas qu' on la porte; mais on en est issu». Famille et belle famille, en effet, donnent lieu à des relations différenciées selon les individus membres. Ego explique qu'elle a beaucoup aidé sa sœur aînée au moment où l'ami de cette dernière l'a abandonnée avec deux enfants en bas âge. Ego qui, à l'époque, s'est alors substituée aux parents catholiques intransigeants, a gardé avec cette sæur une relation qui ne se compare en rien avec celle qu' elle peut avoir avec la troisième sœur, qu' elle voit peu et dont elle ne partage pas les idées.

La fusion est une autre attitude que celle de l'individualisation des relations, mais elle contribue aussi à effacer la distinction entre amitié et germanité. Ego (femme, 42 ans, dentiste) n'a su qu'à 18 ans que celle qu' elle pensait être sa mère n'était que sa belle-mère. Du même coup, ses sæurs sont devenues ses demisœurs. Du côté du conjoint, il n'y a aussi que des sœurs: deux plus âgées, d'un premier lit de la mère et une sœur plus jeune. Les deux fratries sont caractérisées par de forts écarts d'âge, de grands éloignements géographiques (une sœur habite au Canada, une autre en Suisse), de grands écarts socio-économiques (Ego et son conjoint, dentiste lui aussi, ont un cabinet dentaire prospère; les autres sœurs ont des situations modestes ou précaires).

Le couple qui a de nombreux amis aime être entouré. Comme le dit Ego: «J'adore inviter plein de monde». Des deux côtés, les père et mère sont encore en vie et apprécient des réunions de familles d'un style différent. Du côté du conjoint où l'on a toujours beaucoup voyagé, les photos de ces déplacements sont au centre des réunions. De l'autre côté, les réunions de familles se font à l'occasion de sorties culturelles (exposition de peinture ou visite en groupe du Mont Saint Michel). Se mélangent alors les amis des père et mère d'Ego et les enfants qui le veulent. Il arrive aussi au couple d'Ego d'inviter ensemble les parents des deux côtés. Les meilleurs amis ne sont pas moins chers que les membres de la fratrie: "A la limite, les amis font partie de la famille, enfin dans notre cour, c' est pareil, ils sont très importants». 
Le volume des relations est un objectif que le «réseauteur» ne néglige pas. Dans l'exemple suivant, il renoue avec la fratrie après avoir rompu à la fin de l'adolescence. La personne Ego (homme, 43 ans, animateur socioculturel) a quitté sa famille à la fin de l'adolescence, à la mort de son père. Il n'a pas supporté les comportements de ses frères aînés qui ont cherché à prendre la place du père, une fois celui-ci disparu. Le temps s'est écoulé. Ses sœurs ont activement repris la gestion de la fratrie quand la mère a décliné (7 enfants et d'origine polonaise). Ego se rapproche du groupe avec lequel il avait rompu jadis. Il apprécie cependant de garder une certaine distance avec sa fratrie: "Mon épouse et moi nous avons tous les deux une relation à la famille qui est un peu étrange. A la fois on est content de voir les membres de notre famille et en même temps on est bien content de ne pas les voir. Ouais!, quand il y a un repas où l'on est 25 personnes, c' est bien qu' il $y$ en ait un tous les deux ans».

La qualité des relations est une seconde dimension pour laquelle le «réseauteur» n'a pas les mêmes a priori que le séparateur. Il lui arrive de se confier à un frère ou à une sœur. Celui-ci ou celle-ci est alors plus un ami qu'un frère ou une sœur. Les relations avec les amis peuvent être plus permanentes que celles avec ses propres germains. Ego (homme, 34 ans, animateur socioculturel), divorcé et remis en couple avec V.., dit n'avoir pas du tout l' esprit de famille. Il est fâché avec son frère. Depuis qu' est née sa seconde fille, il a repris contact avec sa sæur plus âgée mais tout en gardant ses distances. Il est beaucoup plus tourné vers les amis. Il a fait récemment construire sa maison dans un lotissement où sont aussi installés ses copains du foot. Tous s'entraident et bricolent les uns pour les autres. Cependant, il ne confond pas ces relations de copinage avec l'amitié.

Au cours de sa vie, Ego a rencontré et conservé des relations avec trois personnes qui vivent loin de lui maintenant et qui ne se connaissent pas entre elles. "Les amis, moi j' en ai trois (...) Alors que les copains, tu peux en avoir 10.000. (..) Y en a un, c'est plus que mon frère. On n'a pas été élevé ensemble, mais on s'est bien connu à l'adolescence et depuis, quoiqu' il arrive, tout se sait. C'est à lui que j' ai présenté en premier mon amie V., avant ma famille. L'autre, celui que je vais voir à saint Jean de Luz, je l'ai rencontré il y a dix ans, quand je bossais. Il s' est passé quelque chose de très fort, mais je ne sais pas comment l' expliquer. Avec mon ami d'adolescence, non plus, on n' a pas eu du tout la même vie. (...) Ce sont des personnes sur qui je peux compter pour n' importe quoi».

Le réseau relationnel, quelles que soient ses composantes, amis ou germains, est conçu comme un instrument utile, notamment sur le plan économique. Par exemple, les fêtes organisées par le beau-frère peuvent être utiles pour la recherche d'un emploi. Ego (homme, 40 ans, cadre au chômage) se replie sur son groupe d'adolescence, lorsqu' il est rejeté par sa mère et ses sœurs, après la mort de son père. Ego a épousé la sœur de ses amis rencontrés pendant ses études supérieures à Paris (ses père et mère habitaient dans le midi). Il n'a pas été bien accueilli par les parents de sa femme. Il a haussé le ton et remis son beau-père à sa place, un an avant d'épouser cette dernière. Ce haut fait lui vaut un certain prestige auprès de ses beaux-frères qui se sont ensuite sentis plus libres de rompre avec leurs propres parents. L'un vit encore en Amérique au moment de l'enquête. Ego fréquente l'autre beau-frère mais aussi les amis artistes de ce dernier. Il ne désespère pas de faire des rencontres utiles dans ce milieu et de sortir ainsi de ses difficultés d' emploi actuelles. 
Le réseau relationnel est géré comme un patrimoine, un capital, en tenant compte du fait qu'on ne peut jamais savoir à l'avance quelles sont les relations qui se révèleront «rentables». La prévoyance peut s'exercer pour soi mais aussi pour ses descendants. Ego (un homme) gère ses relations un peu comme un portefeuille. D'abord, il a un noyau d'amis qui date de ses études supérieures à Sciences-po Paris. Sa première femme, $n$ ' ayant jamais réussi à appartenir à cette clique, l'a quitté. Sa seconde femme y a été acceptée comme membre et maintenant contribue à en changer la composition. Ensuite, il reste en contact avec un de ses cousins qui vit à l'étranger et qu'il considère comme le frère qu'il n'a pas. Il entretient aussi les liens avec la famille éloignée, par exemple, en participant bénévolement au débroussaillage d'une propriété qui ne lui appartient pas mais qui est considérée comme la maison de famille. Enfin, il ne s'entend pas bien avec sa sæur qui fait une carrière brillante dans la gestion culturelle mais a une vie privée chaotique. Il fait ce qu' il faut néanmoins pour maintenir les liens avec elle, car il ne veut pas enlever à son fils la possibilité d'avoir des liens forts avec sa seule cousine germaine. Il semble, en effet, que cet objectif intergénérationnel fasse bien partie des buts principaux qu'il assigne à sa vie sociale et familiale.

En résumé, les « réseauteurs » ont des comportements paradoxaux au regard des définitions classiques de l'amitié et de la germanité, telles qu'on les retrouve parmi les séparateurs. Ils ont des relations d'amitié qui peuvent être indestructibles. Certaines de leurs relations de germanité sont de même nature que celles de l'amitié profonde: elles peuvent prendre un tour passionnel et entraîner des ruptures. A la différence des séparateurs, les «réseauteurs » ne considèrent donc pas les germains et les amis comme appartenant à deux classes distinctes. Ils sont aussi éloignés du groupe des pairs dans la gestion de leurs relations. Ils ne cherchent pas d'approbation dans le recrutement de leurs amis et ne se soumettent pas à une ligne de conduite dans les actions coordonnées qu'ils peuvent entreprendre avec les uns et les autres (amis ou germains). Et s'ils appartiennent à des cercles, aucun d'eux n'exerce une influence prépondérante sur leur vie sociale. Ils développent une «gestion prévisionnelle » de la germanité et de l'amitié qui ne s'embarrasse d'aucun a priori et fait feu de tout bois. Des frères et sœurs ou des amis, on ne sait pas ex ante qui remplira le mieux les diverses fonctions de la vie sociale et notamment les fonctions d'assurance et celles liées à la confidence et à l'intersubjectivité.

\section{CONCLUSION}

Les lois sur la famille et sur le couple précisent les droits et les devoirs qui en dérivent pour la filiation et l'alliance. Mais, dans les sociétés occidentales modernes, la germanité et l'amitié, ne sont pas soumises au système juridique (ou de façon très marginale pour les germains à travers l'obligation alimentaire). Les prestations échangées dans ce cadre ne sont pas, non plus, de type contractuel. Une régulation sociale des échanges existe néanmoins, mais elle est informelle et surtout, comme nous venons de le voir, elle n'est pas uniforme.

Le groupe des pairs constitue une communauté exclusive au sein de laquelle les rapports ne font pas de différence entre les amis et les germains. Chacun, du moment qu'il en est membre, attend la même entraide de tous et offre à tous la même entraide. Le sociologue, recourant à la formulation durkheimienne, qualifiera cette 
solidarité de mécanique. Les séparateurs agissent différemment. Leur entraide obéit à un principe de différenciation fonctionnelle. Ce ne sont pas les mêmes prestations que la personne doit attendre des amis et des germains et qu'elle doit leur offrir. Les deux type de relations interpersonnelles renvoient à des fondements différents et les réseaux ne sont jamais confondus. La solidarité peut alors être qualifiée d'organique. Les «réseauteurs » construisent encore autrement les relations d'entraide. Ils recrutent et gèrent leur réseau personnel comme un capital, en utilitaristes, selon le principe: «Je peux t'être utile, tu peux m'être utile». En l'absence de régulation normative, que vaut cet engagement? L'entourage - frères et sœurs ou amis - n'est pas habilité à faire pression en cas de défection. Chacun alors ne compte que sur ses ressources individuelles pour inciter l'autre à donner (ou à se donner) et amorcer ainsi le processus de réciprocité. Les «réseauteurs » ignorent les intérêts collectifs du groupe qu'ils forment avec leurs amis ou leurs germains.

La solidarité désigne chez Durkheim l'interdépendance des parties d'un ensemble. Mais, comme le note Philippe Besnard (1993), commentant De la division du travail social (1893), à cette idée de cohésion se mêlent des considérations morales, en particulier lorsque la solidarité est de type organique: c'est par la réglementation morale que la solidarité «prédétermine » la manière dont les différents organes doivent habituellement concourir. Faute de quoi, il y a anomie et pathologie sociale.

Nul doute que Durkheim aurait considéré le modèle d'entraide des « réseauteurs » comme une pathologie, un système de relations et d'échanges dépourvu de toute forme de régulation normative. Or, Ph. Besnard (ibid.) a attiré l'attention sur le fait que «les pathologies des sociétés modernes» identifiées par Durkheim n'ont pas disparu, plus d'un siècle après la publication de son ouvrage, «tant ces formes anormales paraissent être la normalité des sociétés modernes ». De deux choses l'une: soit, on considère que la fréquence est gage de normalité et de fonctionnalité, le terme pathologique devant être abandonné; soit, on dissocie normalité statistique et pathologie sociale sur la base d'un idéal du lien social qui fait de la réglementation morale un requisit nécessaire. Si Durkheim a constamment louvoyé entre ces deux options, il apparaît clairement aujourd'hui que la seconde est désuète en ce qu'elle ne prend pas la mesure de la formidable promotion de l'individu au cours des cent dernières années. D'autres formes de solidarité à la régulation moins normative ont vu le jour.

Ce constat vaut tout particulièrement pour les comportements entre germains et entre amis. Au sein des sociétés occidentales modernes existe une forme de solidarité entre proches - amis ou germains -, celle des «réseauteurs » qui est largement répandue, fonctionnelle, et qui, pour ces deux raisons, ne mérite pas d'être qualifiée de pathologique. Diagnostiquer, comme J. Coleman (1990) au sujet du capital social, un déclin des structures sociales «primordiales » liées à la famille et aux relations entre proches, semble bien imprudent. D'autant que les différents modèles d'entraide sont cumulatifs: l'émergence de formes nouvelles n'implique pas la disparition concomitante des formes antérieures. Tout se conserve.

Observatoire Sociologique du Changement (OSC)

Fondation nationale des Sciences Politiques, Paris

jeanh.dechaux@sciences-po.fr

nicolas.herpin@insee.fr 


\section{RÉFÉRENCES BIBLIOGRAPHIQUES}

Besnard Ph., Les pathologies des sociétés modernes, in Ph. Besnard, M. Borlandi, P. Vogt (dir.), Division du travail et lien social. Durkheim un siècle après, Paris, PUF, 1993.

Coleman J. S., Foundations of social theories, Cambridge, Belknap Press of Harvard University Press, 1990.

Crenner E., Famille, je vous aide, INSEE-Première, $\mathrm{n}^{\circ}$ 631, 1999.

Déchaux J.-H., Le souvenir des morts. Essai sur le lien de filiation, Paris, PUF, 1997.

Gans H., The Urban villagers, New York, Free Press, 1962.

Habermas J., «La modernité: un projet inachevé», Critique, n 413, 1981. 